\title{
Treatment of a fibular autograft non-union with a resulting deformity by stabilization, progressive correction and callotasis using an Ilizarov fixator: a case study
}

\author{
Yasser Elbatrawy • Giby C. Philips
}

Received: 11 May 2008/Accepted: 12 March 2011/Published online: 12 April 2011

(C) The Author(s) 2011. This article is published with open access at Springerlink.com

\begin{abstract}
Bone tumours present a challenge to reconstructive surgery when the tumour breaches the physeal and periphyseal region of the growing bone. Though a host of options are available, these are not without complications. We report one such case of osteosarcoma of the tibia treated initially with wide resection of the tumour and intercalary fibular strut grafting using plate and screws. The operation was complicated by a non-union at the proximal tibio-fibular autograft junction. This leads to a multiplanar deformity with severe procurvatum at the proximal tibio-fibular graft junction, which was successfully treated by callotasis using an Ilizarov fixator. Appropriate consent was obtained from the patient and parents to publish this case report.
\end{abstract}

Keywords Ilizarov · Tumour · Graft · Osteosarcoma · Bone defect $\cdot$ Reconstruction - Deformity correction

\section{Case report}

A 13-year-old boy was diagnosed with osteosarcoma of the proximal third of his right tibia in 2003. The surgical intervention comprised of a wide sub-physeal excision with safe margin, followed by autogenous ipsilateral fibular strut grafting. The tibio-fibular synostosis bridging the segmental bone gap was augmented with buttress plate and screw fixation.

\section{Y. Elbatrawy}

Elzahra'a University Hospital,

Azhar University, Cairo 11884, Egypt

e-mail: yasser@elbatrawy.com

G. C. Philips ( $\square)$

Welcare Hospital, Cochin 682019, Kerala, India

e-mail: docgiby@hotmail.com
The boy presented to us 4 years after the initial surgery, with a flexion (procurvatum) deformity just below the level of the knee (Figs. 1, 2). The deformity was a result of a non-union at the proximal pole of the autograft. On clinical evaluation, the active range of motion was $40^{\circ}-120^{\circ}$ with minimal mobility at the non-union site. The severity of the deformity had restricted ambulation to partial weight bearing using crutches.

Pre-operative radiographic assessment of the mechanical axis of the tibia showed the deformity was predominantly in the saggital plane. The centre of angulation of the deformity (CORA) was seen to coincide with the proximal tibio-fibular graft junction. The mechanical axis in the anteroposterior (AP) view fell within the normal range, making the deformity uniplanar in nature and requiring correction in the saggital plane alone. On lateral view, the apex of the deformity coincided with the proximal tibio-fibular graft junction, which appeared to have not fused completely at the time. This was confirmed clinically also. The apex was also found to lie within a centimetre of the open physeal plate. Though the buttress plate and screws appeared intact, it was easily discerned that one of the proximal screws had inadvertently crossed the physeal plate. A Doppler study was done to ascertain the vascularity of the fibula as well as the leg distally. The leg was fully sensate distally. However, after careful consideration of the pros and cons, open surgery for acute correction of the deformity was ruled out. Based on the X-ray findings, it appeared possible to address the deformity correction with the plate and screws in situ and without disturbing the soft tissue biology. An Ilizarov fixator spanning the grafted fibular, comprising of Schantz screws and k-wires, was planned, in accordance with the hybrid advanced technique of Ilizarov fixation. 


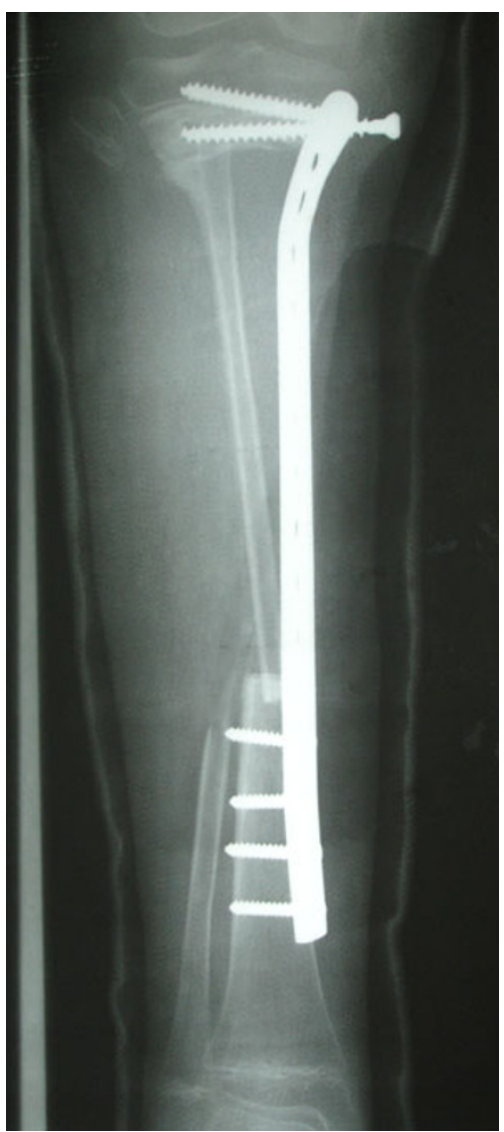

Fig. 1 AP view showing the fibular graft reconstruction with physeal breach and mechanical failure of the proximal screws

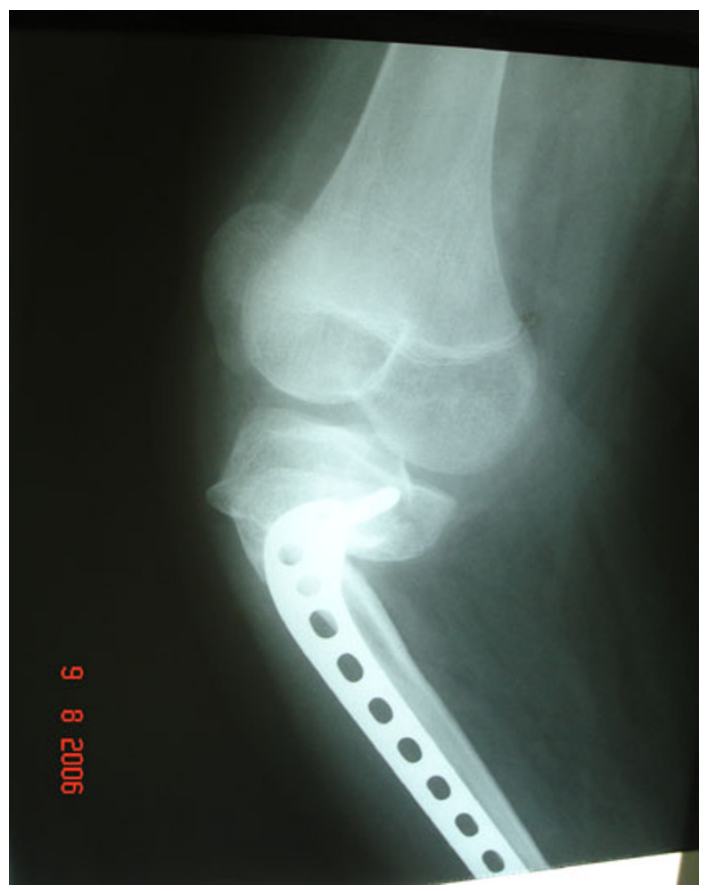

Fig. 2 Lateral view with marked procurvatum deformity
In the operating room, a 3-ring hinged Ilizarov frame was pre-assembled for application. The CORA was determined and marked under fluoroscopy. We began by removing the errant screw in the physis through a key-hole incision. Physeodesis was carefully carried out using a 2-mm drill bit through 2 separate medial and lateral miniincisions. The plate and remaining screws were left undisturbed. Next, using the standard approach for fixation, the pre-assembled frame was mounted and centred using $\mathrm{k}$-wires. The two hinges on the proximal rings were aligned to correspond to the CORA in the saggital plane. Care was taken to align the hinges parallel to the long axis of the plate screws. This would allow the correction to occur at the apex of the deformity without disturbing the proximal screw. In turn, the proximal screw would behave like a fulcrum allowing rotation, and thereby correction in the saggital plane. The frame was then extended to the femur to augment the fixation, the sub-epiphyseal bone stock being inadequate for a rigid fixation using Shantz screws alone. In addition, the knee-spanning femoral rings locked the knee joint, to make it behave as a single unit (longer lever arm) with the femur proximally. With such a configuration of the construct, angular correction would occur only along the hinges, i.e. the CORA of the deformity (Fig. 3).

The correction was started on the first post-operative day, the rate of correction set to correct $1^{\circ}$ per day. Full correction was attained on the 40th post-operative day, when the mechanical axis of the tibial was checked for the alignment. Slight distraction at the CORA was initiated at full angular correction. This was aimed at enhancing the rate of osteogenesis at the non-united site, as well as, widens the area of regenerate by callotasis to achieve a stronger tibio-fibular synostosis. Partial weight bearing was started once the angulation was corrected. Serial X-rays were taken at every 2 weeks, and a final X-ray taken at the end of correction, i.e. when the rings were parallel to each other, emperically denoting end of correction. Dynamization was done 1 month prior to frame removal and the leg put in a long leg cast for 5 weeks. The patient was discharged with strict advice against contact sports and heavy activity (for 1.5-2 years) as well as regular follow-ups to monitor graft union.

In this case, the frame was dynamized 11 weeks out and the frame was removed at the end of 15 weeks. The range of motion at 6 months post-frame removal was $0^{\circ}-110^{\circ}$ (Fig. 4a-c).

\section{Discussion}

The surgical treatment of malignant bone tumours such as osteogenic sarcoma requires wide resection of the involved 
Ilizarov frame construct with the hinges at the level of the proximal tibio-fibular junction in the frontal plane. b Lateral view of the Ilizarov frame construct with the hinges at the CORA of the deformity in the saggital plane
Fig. 3 a AP view of the
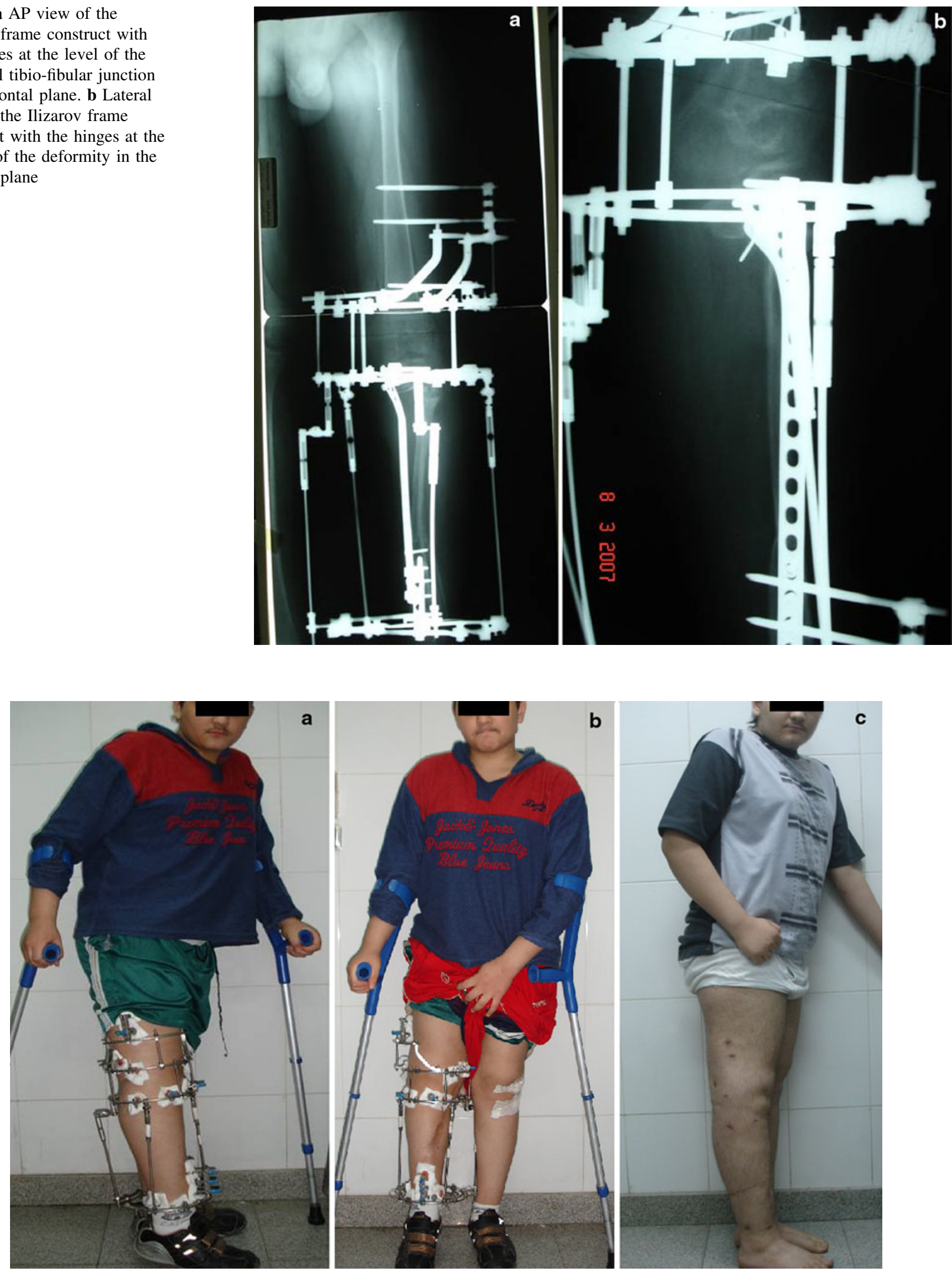

Fig. 4 a Alignment achieved in frame at end of correction (side view). b Alignment achieved in frame at end of correction (front view). c Ambulatory limb alignment out of frame 
bone. The use of corticocancellous bone grafts, vascularized/non-vascularized fibular grafts and bone transport for bridging wide defects in the bone is well documented [13]. However, it is case specific and depends on a host of factors ranging from surgeon/patient preference, length of treatment, associated potential for complications to monetary considerations. Amputations remain the last resort for most patients.

Autologous iliac crest grafts have historically been used to fill segmental bone defects, but constraints relating to the amount of graft and donor site morbidities associated with larger grafts make it less favourable in situations of massive bone loss $[1,4]$. Both free vascularized fibular grafts and ipsilateral vascularized fibular transfer (IVFT) are used in larger defects of more than $6 \mathrm{~cm}$ [4-9]. The free vascularized fibular flap transfer provides for a good cortical bone graft of up to $25 \mathrm{~cm}$ in length with a good vascular pedicle [10], but it requires a skilled microvascular team, lengthy operative time, post-operative monitoring and the potential for complications such as donor site pain, delayed wound healing and sensory deficits [10]. Bone transport can be used both in small and large tibial defects as a definitive primary treatment option without the use of microsurgical techniques [1,2]. In this case report, the Huntingtons modification of the fibula-pro-tibia technique was used to achieve a tibio-fibular synostosis [8, 11-14].

The fibula being the strongest autogenous bone graft available with minimal donor site morbidity made it a natural choice for reconstruction in this patient $[15,16]$. Yadav et al. have reported the use of dual fibular grafting for massive bone gaps in the lower extremity.

The surgical treatment in our case study involved resection of the tumour and bridging of the gap with a nonvascularized ipsilateral fibular graft. The ensuing complication of fixed flexion deformity resulted from a mobile non-union. An effective management warranted the removal of misplaced hardware, correction of angulation and effective stabilization of the correction. All surgical options were carefully considered. The option of a revision ORIF and corrective osteotomy entailed further soft tissue compromise and potential risk of non-union and infection, which could be disastrous. In such instances, the integrity of the soft tissue envelope out-weight mechanical stability in maintaining fixation $[17,18]$. Ilizarov surgery was an option based on the finding from earlier studies that distraction of a hypertrophic non-union leads to union. The versatility of the Ilizarov fixator in effectively correction the deformity without the need to reopen the surgical site, whereby minimizing the chances of infection and nonunion, made it an attractive alternative. The patient would also be allowed early weight bearing after full correction was achieved, as opposed to an acute corrective osteotomy and reapplication of a bridge plate. Further, the affected limb looked osteoportic on radiography, which was largely borne out of disuse. Using the Ilizarov device in such a situation would help reestablish early weight bearing status to the limb and enhance healing.

Though the Ilizarov technique may yield good results in the hands of someone well versed in the techniques, the potential complications include pin track infections, docking site non-union and prolonged treatment time, which may include several trips to the OR $[1,19,20]$.

\section{Conclusion}

This case report demonstrates the Ilizarov approach to treating a case of deformity and shortening, arising from a mobile non-union around the proximal physis of the tibia. In such a situation, the versatility and minimal invasive benefits of an Ilizarov fixator make it a preferred choice of treatment for deformity correction and/or bone union, in the hands of one proficient in the technique.

This makes the Ilizarov fixator and distraction osteogenesis a useful adjuvant in treating such cases.

Open Access This article is distributed under the terms of the Creative Commons Attribution License which permits any use, distribution and reproduction in any medium, provided the original author(s) and source are credited.

\section{References}

1. Green SA (1994) Skeletal defects. a comparison of bone grafting and bone transport for segmental skeletal defects. Clin Orthop Relat Res 301:111-117

2. Christian EP, Bosse MJ, Robb G (1989) Reconstruction of large disphyseal defects, without free fibular transfer, in grade 111-B tibial fractures. J Bone Joint Surg Am 71:994-1004

3. Weiland AJ (1981) Current concepts review: vascularized free bone transplants. J Bone Joint Surg Am 63:166-169

4. DeCoster TA, Gehlert RJ, Mikola EA, Pirela-Cruz MA (2004) Management of posttraumatic segmental bone defects. J Am Acad Orthop Surg 12(1):28-38

5. Weiland AJ (1984) Vascularized bone transfers. Instr Course Lect 33:446-460

6. Sowa DT, Weiland AJ (1987) Clinical applications of vascularized bone autografts. Orthop Clin North Am 18:257-273

7. Brown KL (1991) Limb reconstruction with vascularized fibular grafts after bone tumor resection. Clin Orthop Relat Res 262:64-73

8. Hertel R, Pisan M, Jacob RP (1995) Use of the ipsilateral vascularized fibula for tibial reconstruction. J Bone Joint Surg Br 77:914-919

9. Chacha PB, Ahmad M, Daruwalla JS (1981) Vascular pedicle graft of the ipsilateral fibula for non-union of the tibia with a large defect. an experimental and clinical study. J Bone Joint Surg Br 63-B:244-253

10. Koulouvaris P, Theos C, Kottakis S, Demertzis N (2007) A simple treatment for a $15 \mathrm{~cm}$ tibia bone defect: a case report of an ipsilateral vascularized fibula transfer. J Orthop Trauma 21(3):215-218 
11. Agiza AR (1981) Treatement if tibial osteomyelitic defects and infected pseudoarthrosis by the Huntington fibular transference operation. J Bone Joint Surg Am 63:814-819

12. Kassab M, Samaha C, Saillant G (2003) Ipsilateral fibular transposition in tibial nonunion using Huntington procedure: a 12 year follow-up study. Injury 34(10):770-775

13. Mohler DG, Yaszay B, Hong R, Wera G (2003) Intercalary tibial allografts following tumor resection: the role of fibular centralization. Orthopedics 26(6):631-637

14. Shapiro MS, Endrizzi DP, Cannon RM, Dick HM (1993) Treatment of tibial defects and nonunions using ipsilateral vascularized fibular transposition. Clin Orthop Relat Res 296:207-212

15. Springfield D (1996) Autograft reconstructions. Orthop Clin North Am 27(3):483-492

16. Lee EH, Goh JC, Helm R, Pho RW (1990) Donor site morbidity following resection of the fibula. $\mathrm{J}$ Bone Joint Surg $\mathrm{Br}$ 72(1):129-131
17. Mooney V, Nickel VL, Harvey JP Jr, Snelson R (1970) Castbrace treatment for fractures of the distal part of the femur. a prospective controlled study of one hundred and fifty patients. J Bone Joint Surg Am 52(8):1563-1578

18. Ostrum RF, Geel C (1995) Indirect reduction and internal fixation of supracondylar femur fractures without bone graft. J Orthop Trauma 9(4):278-284

19. Aronson J (1997) Limb-lengthening, skeletal reconstruction, and bone transport with the Ilizarov method. J Bone Joint Surg Am 79(8):1243-1258

20. Cierny G III, Zorn KE (1994) Segmental tibial defects. comparing conventional and Ilizarov methodologies. Clin Orthop Relat Res 301:118-123 